\title{
Implementation of bending-active elements in kinematic form-active structures - Part II: Experimental verification
}

\author{
Silke Puystiens, ${ }^{\mathrm{a}, \mathrm{b}, *}$, Maarten Van Craenenbroeck ${ }^{\mathrm{b}}$, Danny Van Hemelrijck ${ }^{\mathrm{a}}$, Wim Van Paepegem ${ }^{\mathrm{c}}$, \\ Marijke Mollaert ${ }^{\mathrm{b}}$, Lars De Laet ${ }^{\mathrm{b}}$
a Department of Mechanics of Materials and Constructions, Vrije Universiteit Brussel, Pleinlaan 2, 1050 Brussels, Belgium
b Department of Architectural Engineering, Vrije Universiteit Brussel, Pleinlaan 2, 1050 Brussels, Belgium
c Department of Materials Science and Engineering, Ghent University, Technologiepark-Zwijnaarde 903, 9052 Zwijnaarde, Belgium \\ * Corresponding author: silke.puystiens@vub.ac.be
}

\begin{abstract}
The integration of bending-active elements in kinematic form-active structures opens new perspectives for the development of transformable architectural applications, introducing new typologies and interesting structural concepts. Structures that combine the use of membrane materials and the concept of active bending are referred to as 'textile hybrids'.

The complex interaction between the bending-active boundary elements and the membrane, but also the inherent high flexibility of both the membrane material and the bending-active elements, however, complicate the structural behaviour of the studied transformable textile hybrid. Therefore, an experimental verification of the structure becomes crucial to validate the results obtained through the numerical analysis and to allow a full understanding of the structural behaviour.

This paper discusses the experimental set-up and investigation of a representative case study and compares the experimental results to the numerically obtained values.
\end{abstract}

Keywords: Tensile fabric structures; Kinematic structures; Active bending; Experimental investigation 


\section{Introduction}

Lightweight and innovative design concepts on the one hand, and adaptability and transformability on the other hand, are gaining interest in today's architecture. When speaking about transformable structures, the material's weight and deformability play an important role. Therefore, lightweight membrane materials, with their inherent high flexibility, are very suitable to combine with transformable principles.

However, existing cases and studies demonstrate that the membrane is often only tensioned in its fully unfolded state, causing instabilities under e.g. wind loading in the intermediate states, which can thus lead to failure of the membrane. Two examples are the large Umbrellas ( $26 \times 26 \mathrm{~m}$ each) for the Medina Haram Piazza in Saudi Arabia and the Convertible Membrane Roof in Switzerland that covers a $50 \mathrm{~m}$ long pedestrian road (see Figure 1).
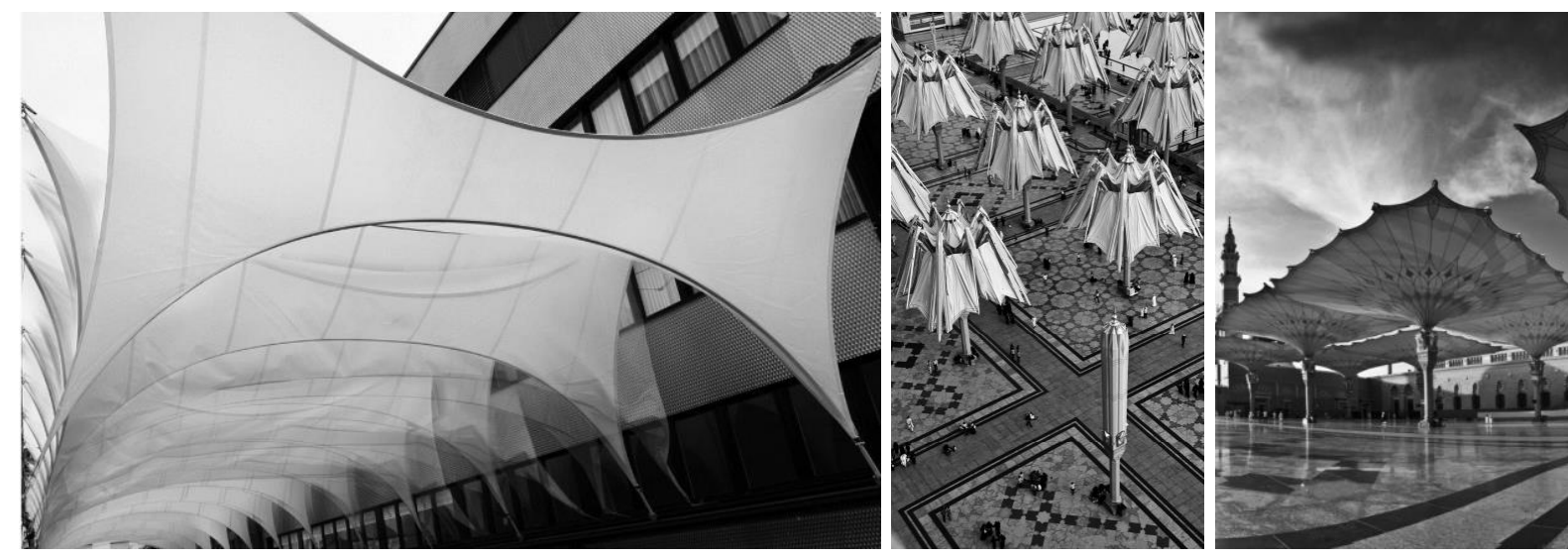

Figure 1. (left) Convertible membrane roof in Switzerland (Kugel Architekten) [1] (middle and right) High-tech deployable umbrellas in Medina (SL Rasch) [2]

Structures combining bending-active elements with prestressed textile elements are now referred to as 'Textile Hybrids'. The inherent flexibility of both bending elements and membranes allows them to create innovative designs and structurally efficient hybrid systems [3].

Moreover, the bending-active elements can provide solutions to keep the membrane tensioned in different phases of a transformation, as is the case in the responsive façade system of the Soft House project in Hamburg (Figure 2). At the top, the membranes are attached to cantilevering bending-active boards that act as compound springs to ensure the preservation of the vertical membrane prestress in all the twisting positions. The horizontal prestress in the membrane strips is maintained by means of bars that push the long edges apart. Also, the use of an uncoated fabric, i.e. a mesh material, contributes to the ability to remain tensioned throughout the different phases of the twisting movement [4].
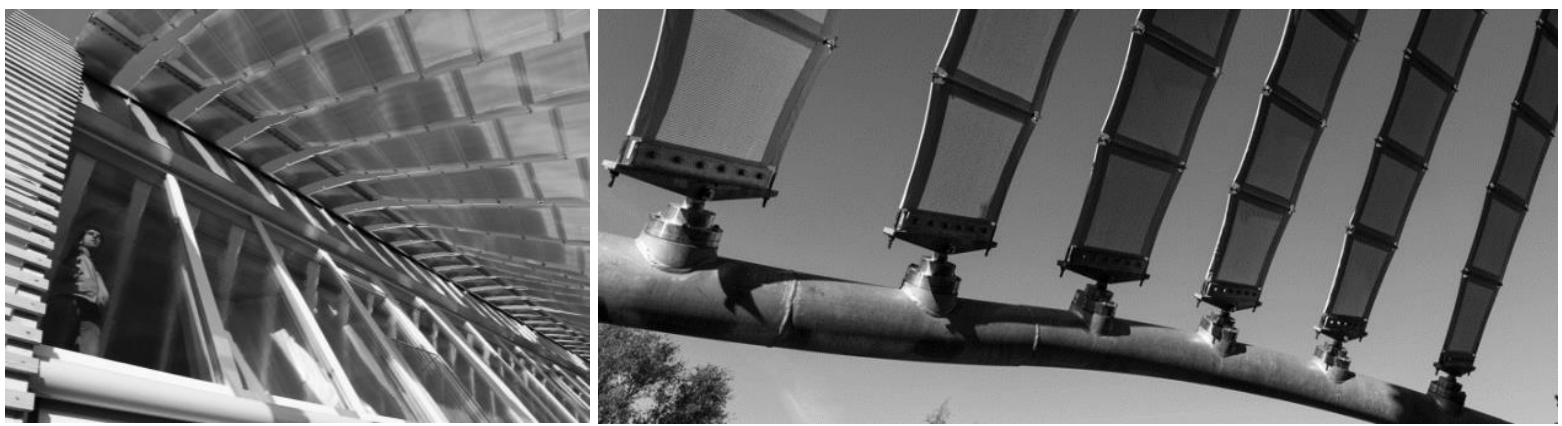

Figure 2. Soft House (IBA): responsive façade system of twisting membrane strips and bending-active GFRP boards (i.e. a textile hybrid) [5] 


\section{Description of the case study}

In this research, a seemingly simple geometry is investigated in order to understand and analyse the response of such a transformable bending-active membrane structure. As described in [6], it concerns a pringle shaped geometry, which is obtained by tensioning a flexible membrane inside a bending-active ring, forming a self-stabilizing system.

Initially, the structure only consists of one bending element with a circular section that is bent towards a ring-shaped geometry (i.e. the 'bending-active ring' in Figure 3) and an initially flat membrane piece. When tensioning the membrane inside the ring, the degree of pretension defines the three-dimensional geometry (i.e. the form-finding geometry). The high points of this pringle-shaped membrane structure are pulled towards each other to achieve the kinematic deployment. The rectangular bending-active beam element, in between these two high points, has proven to improve the structural behaviour during the deployment [6]. Figure 3 illustrates the general principles of the structural behaviour of the transformable pringle-shaped textile hybrid, simulated using the finite element software Sofistik [7]. Figure 4 shows the experimental setup.

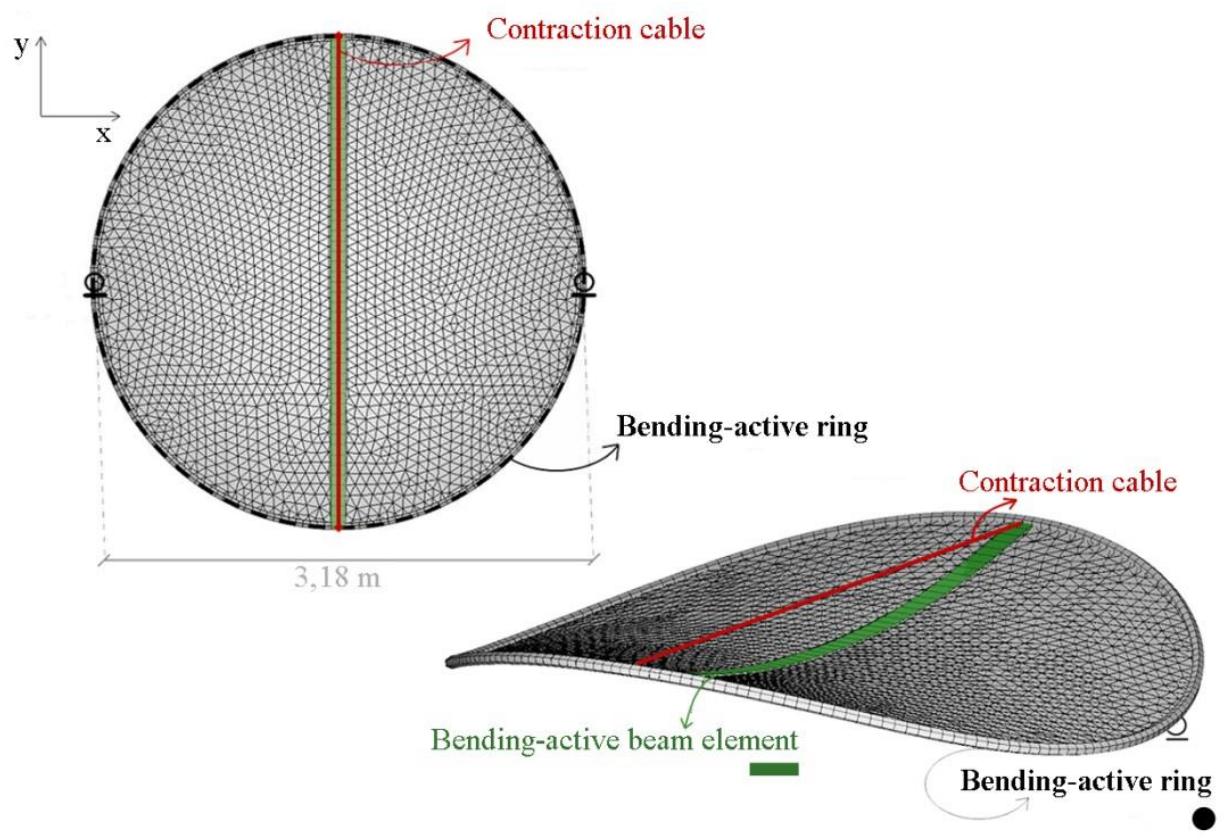

Figure 3. Top view and three-dimensional view of the obtained pringle-shaped textile hybrid, indicating the location of the circular bending-active ring, the rectangular bending-active beam element and the contraction cable

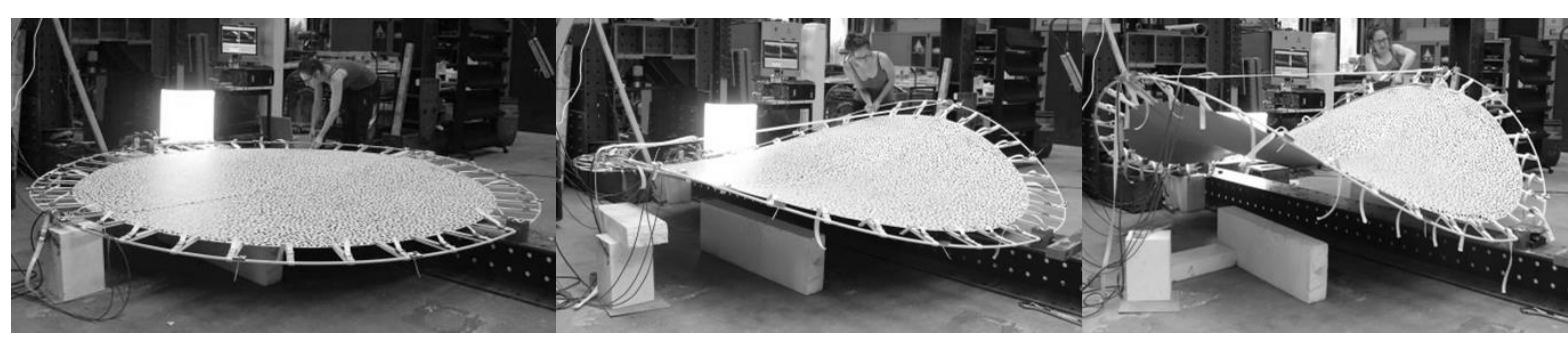

Figure 4. Experimental investigation of the deforming ring element:

(i) flat; (ii) intermediate and (iii) curved position 


\section{Test set-up}

\subsection{Geometry and materialization}

A GFRP rod with a section of $12.7 \mathrm{~mm}$ and a bending modulus of $41 \mathrm{GPa}$ is bent to form a circular ring with a diameter of $3.18 \mathrm{~m}$. To do so, two GFRP rods with a length of $5 \mathrm{~m}$ are glued together with epoxy glue into an aluminium tube (one at each side).

A PU-coated polyester fabric is positioned in the plane of the ring and is attached to the GFRP ring with 40 adjustable polyester belts, to allow gradual tensioning of the membrane. The adjustable links in the experimental set-up are polyester belts with a width of $25 \mathrm{~mm}$, a thickness of $2 \mathrm{~mm}$, an ultimate strength of $28 \mathrm{kN}$ and an approximate linear stiffness EA of $120 \mathrm{kN}(\mathrm{E}=2.4 \mathrm{GPa})$.

The material properties of the PU-coated polyester fabric are presented in Table 1. A number of cruciform specimens are subjected to biaxial tension tests, where after the material parameters are derived following the model of Galliot and Luchsinger [8].

Table 1. Material properties for the PU polyester fabric determined following the model of Galliot and Luchsinger [8]

\begin{tabular}{c|c|c|c|c|c|c} 
& $E_{w}(k N / m)$ & $E_{f}(k N / m)$ & $\Delta_{E w}(k N / m)$ & $\Delta_{E f}(k N / m)$ & $v_{w f}(-)$ & $G_{w f}(k N / m)$ \\
\hline PU PES & 6.65 & 4.56 & 0.00 & 3.72 & 0.49 & 1.52
\end{tabular}

The cutting pattern of the PU membrane is determined through the cutting pattern analysis that is implemented in Sofistik (module TEXTILE). Next to the elastic compensation, i.e. the part accounting for the elastic deformation of the membrane, an additional compensation factor, i.e. the part accounting for the permanent deformation, of $4 \%$ in both membrane directions is applied. Sofistik then returns the cutting pattern presented by the grey line in Figure 5.

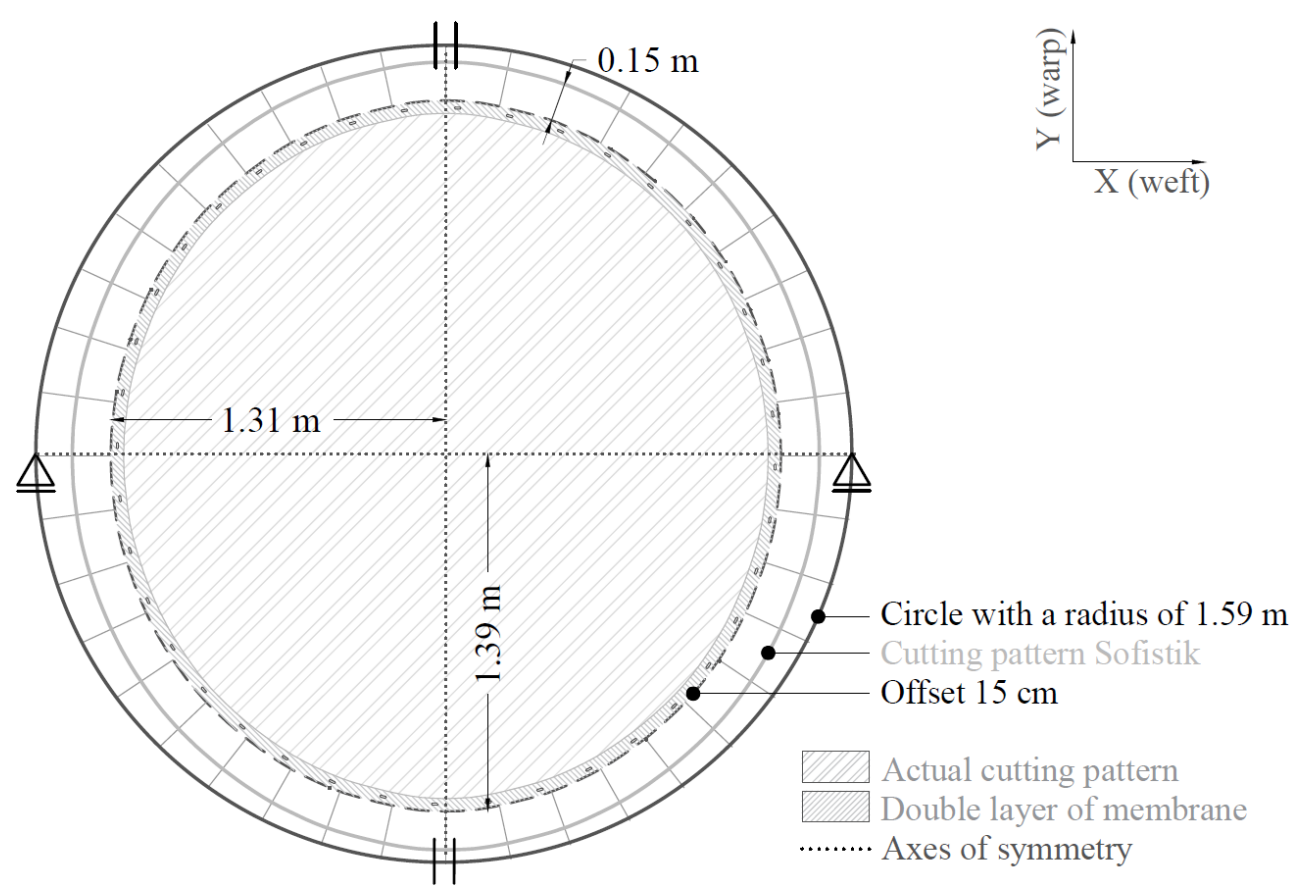

Figure 5. Determination of the actual cutting pattern, based on the generated pattern in Sofistik (= compensation for elastic + permanent deformation), including the polyester belts for the prestressing of the membrane 
For this case, it is decided to generate the membrane as one flat piece of PU-fabric in order to allow a DIC (i.e. Digital Image Correlation [9]) measurement starting from a flat unstressed reference state of the membrane (see section 4.3). To allow the implementation of the tensioning belts, this contour line is offset with $15 \mathrm{~cm}$, resulting in the final cutting pattern that is hatched in Figure 5.

As the PU-coated membrane material provided by Sioen has a width of only $1.45 \mathrm{~m}$, the flat membrane is divided into two parts (see Figure 6) that are welded together with a high frequency welding machine. The connection of the two membrane panels is designed in a way that a pocket is created wherein the internal GFRP beam element (with an E-modulus of $35 \mathrm{GPa}$ ) can be implemented.

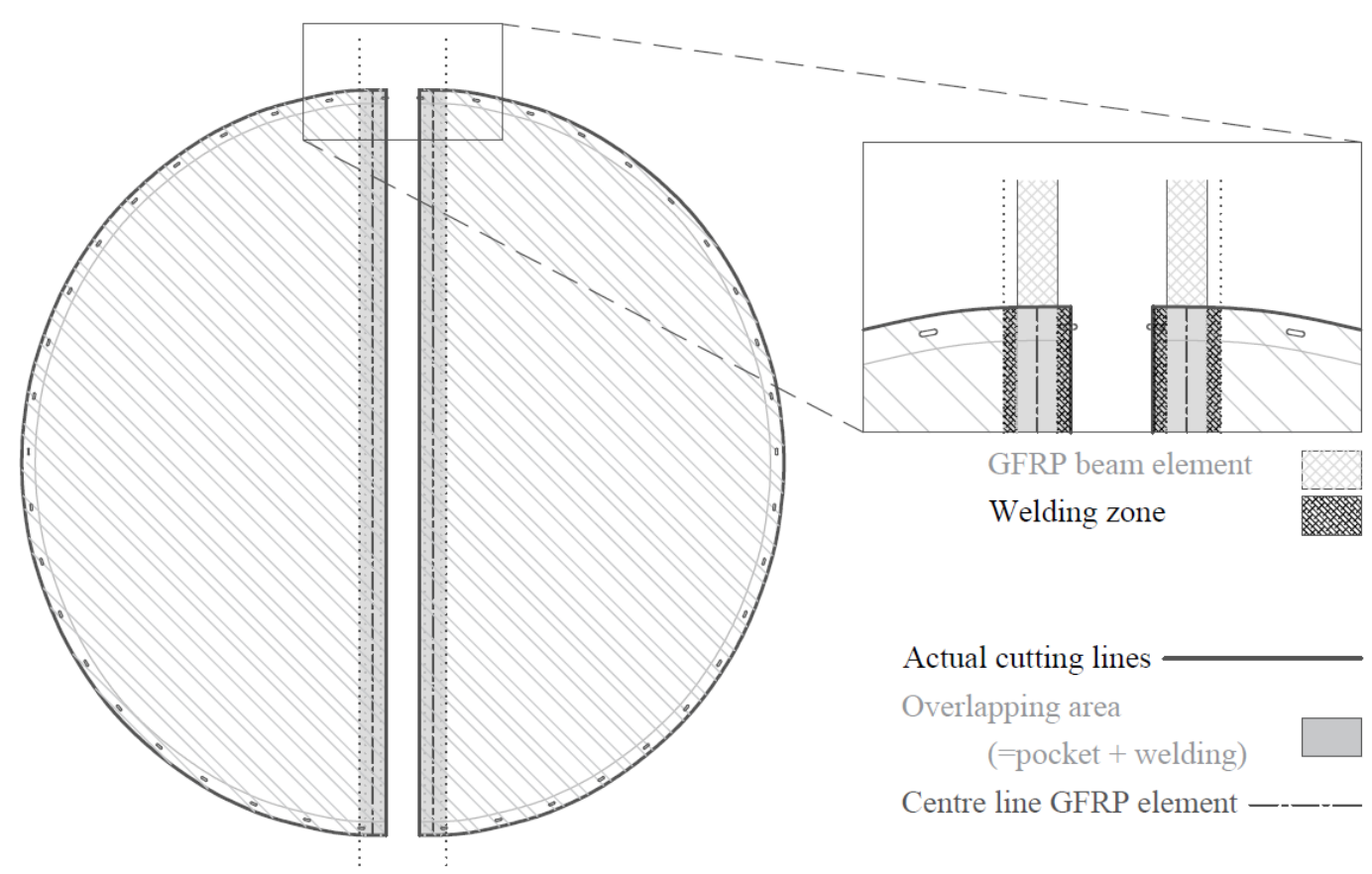

Figure 6. Fabrication detail of the pocket wherein the internal beam element is placed

\subsection{Constraints and connections}

The GFRP ring is placed on 4 supports, of which two can move freely, i.e. the upper points, and two are fixed in the upward direction, i.e. the lower points.

The lower points are clamped in a steel connection that can be fixed onto a steel beam by means of a bolt (Figure 7 (left \& bottom)). One of the lower points is fully fixed during the whole test run, whereas the other point can slide horizontally during the pretensioning of the membrane and is then fixed to perform the kinematic deployment (i.e. when shortening the contraction cable).

At the top points, both the internal beam element and the contraction cable have to be attached (allowing rotation of both elements around the ring), as indicated in Figure 7 (right). 

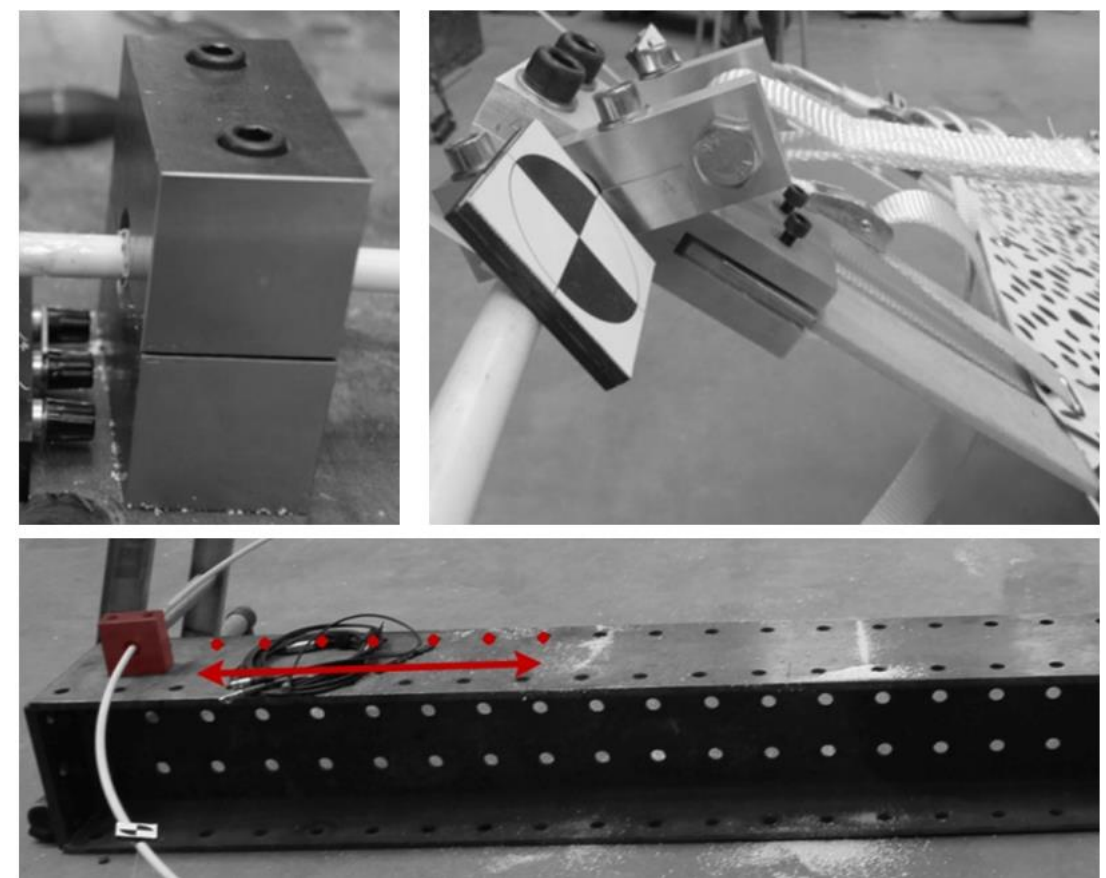

Figure 7. (left) Clamped connection lower points; (right) connection top points; (bottom) one of the clamped supports

\subsection{Introducing the prestress and kinematics}

Along the membrane boundary a second layer of fabric is glued as reinforcement (Figure 5 and Figure 8). Forty oval eyelets are perforated along this reinforced zone, equally spaced to allow a uniform tensioning of the membrane in the GFRP ring. Through these eyelets polyester belts are connected with a small ratchet, as indicated in Figure 8. This allows manual tensioning of the membrane with discrete steps: the length of the belts is measured manually at every step to obtain a more or less equal/symmetrical prestress.

After the membrane is pretensioned in the boundary ring, the kinetic deployment of the system is initiated. This is done by means of shortening the 'contraction cable', which is in this case a polyester belt interconnecting the two top points (Figure 8). Similar to the small belts to apply the pretension, the internal belt is provided with a small ratchet.
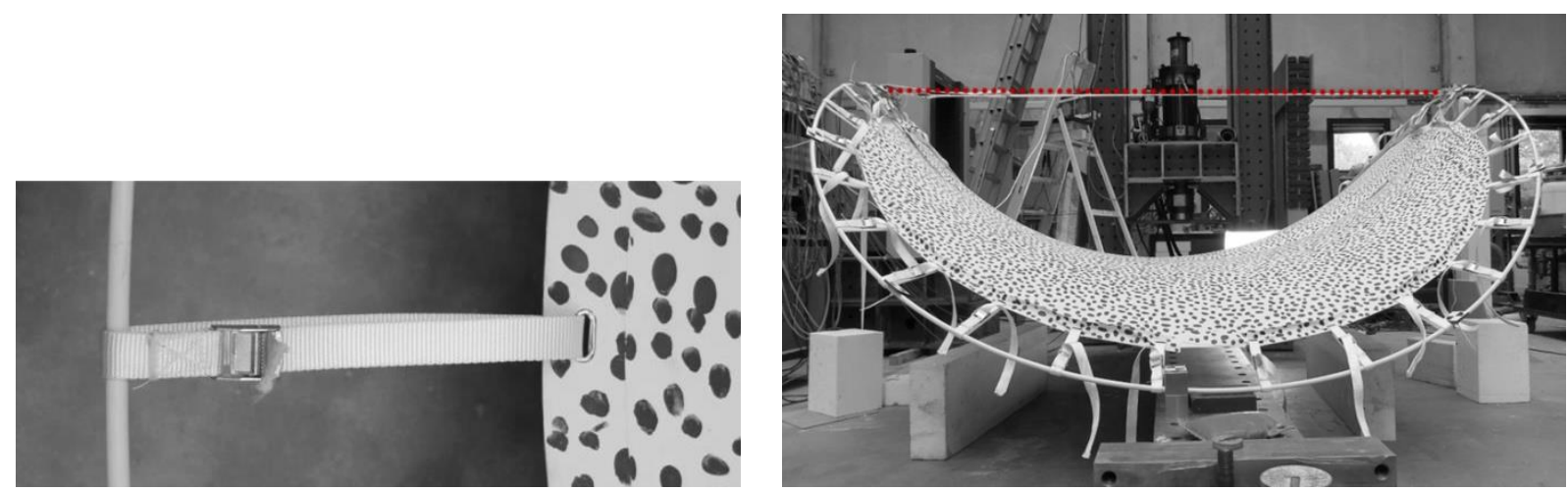

Figure 8. (left) Detail of the adjustable belts connecting the membrane (with speckle pattern) to the bendingactive ring element; (right) Contraction cable between the two upper points, to simulate the kinematic deployment of the membrane structure 


\section{Measurement equipment}

Measurements are done to compare the experimental results with the numerically obtained values, but also to confirm the feasibility of the structural principle.

\subsection{Manual measurements}

The manual measurements are performed using a simple meter and are mainly used to make sure that all adjustable link lengths are approximately uniform in each step of the prestressing process. Furthermore, the structure's overall geometry is verified through manual measurements. Only some crucial lengths are measured in order to validate the lengths that are calculated through the DIC measurements (by means of the tracking of discrete points).

\subsection{Strain gauges}

To determine the stresses in the beam during the transformation, strain gauge rosettes are used. Three rosettes that measure the strains in three directions (at $0^{\circ}, 45^{\circ}$ and $90^{\circ}$ ) are glued onto the beam's surface: (i) on the upper side of the structure's top point, (ii) on the upper side of the beam at the location of the clamped support and (iii) at the bottom of the beam at the location of the clamped support. The measurement of the strains in three directions allows calculating the plane stresses in the two principle directions at each of the three locations. Figure 9 shows the positioning of the strain gauge rosettes as applied in the experimental set-up.
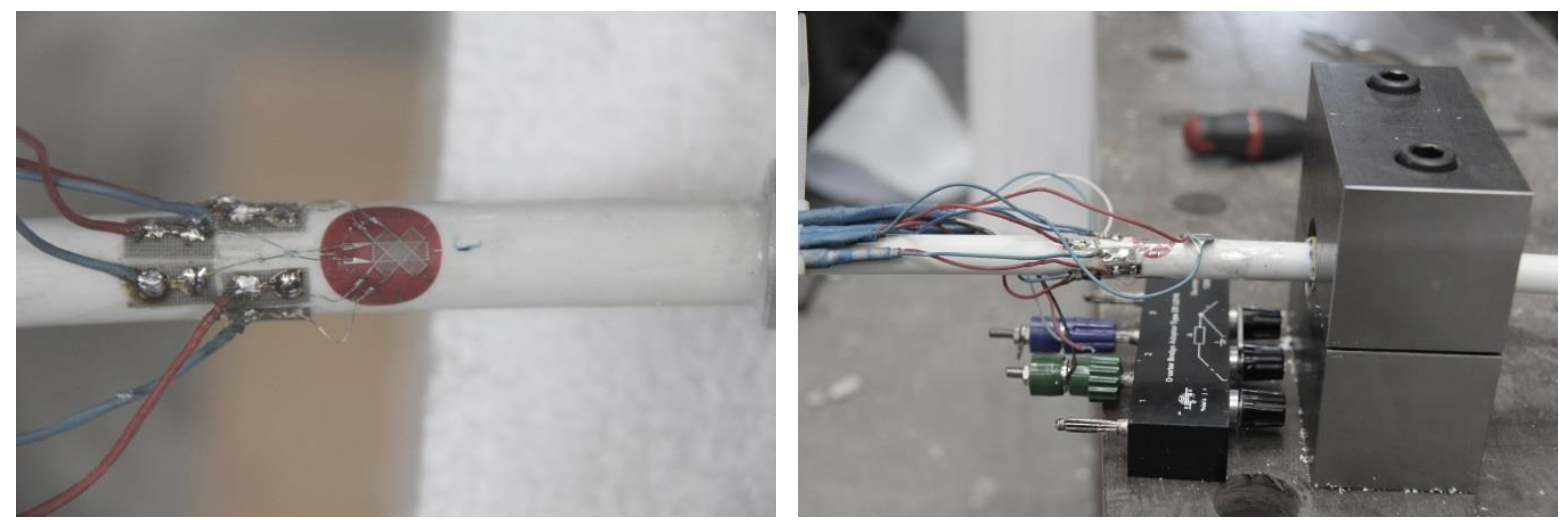

Figure 9. Strain gauge rosettes: (left) one is positioned at one of the top points; (right) two are positioned near to one of the supports (one on the upward and one on the downward side)

\subsection{Digital Image Correlation}

At a height of $4 \mathrm{~m}$ the DIC cameras are attached to a meccano beam at a distance of approximately 3.5 $\mathrm{m}$ to capture the whole structure. Two coupled cameras with $8 \mathrm{~mm} \mathrm{C}$-mount lenses are used, placed under a different angle to allow three-dimensional measurements. More information on the principles and terminology of the DIC system can be found in [9]. The Point Grey Grasshopper3 ccd cameras (type GS3-U3-51S5M, (Point Grey)) have a resolution of 2448 x 2048 pixels.

The correlation of the DIC images is performed with a subset of $27 \times 27$ pixels and a step size of 5 pixels. The filter size for the calculation of the strains is set at 7 , smoothing the strain field over an area of $7 \times 5$ pixels.

The membrane's surface is provided with a speckle pattern to allow a full field measurement (Figure 10 (top)), whereby displacements and strains in the membrane can be visualised throughout the test. 
Furthermore, by placing markers at a number of characteristic points, the geometry of the pringle shape can be tracked automatically throughout the pretensioning and deployment (Figure 10 (left)). In the most important steps of the test process, a series of virtual markers are placed along the ring, the membrane boundary and the internal member (Figure 10 (right)). This not only allows the verification of the overall geometry, but also serves as a verification of the measured length of the pretensioning belts.

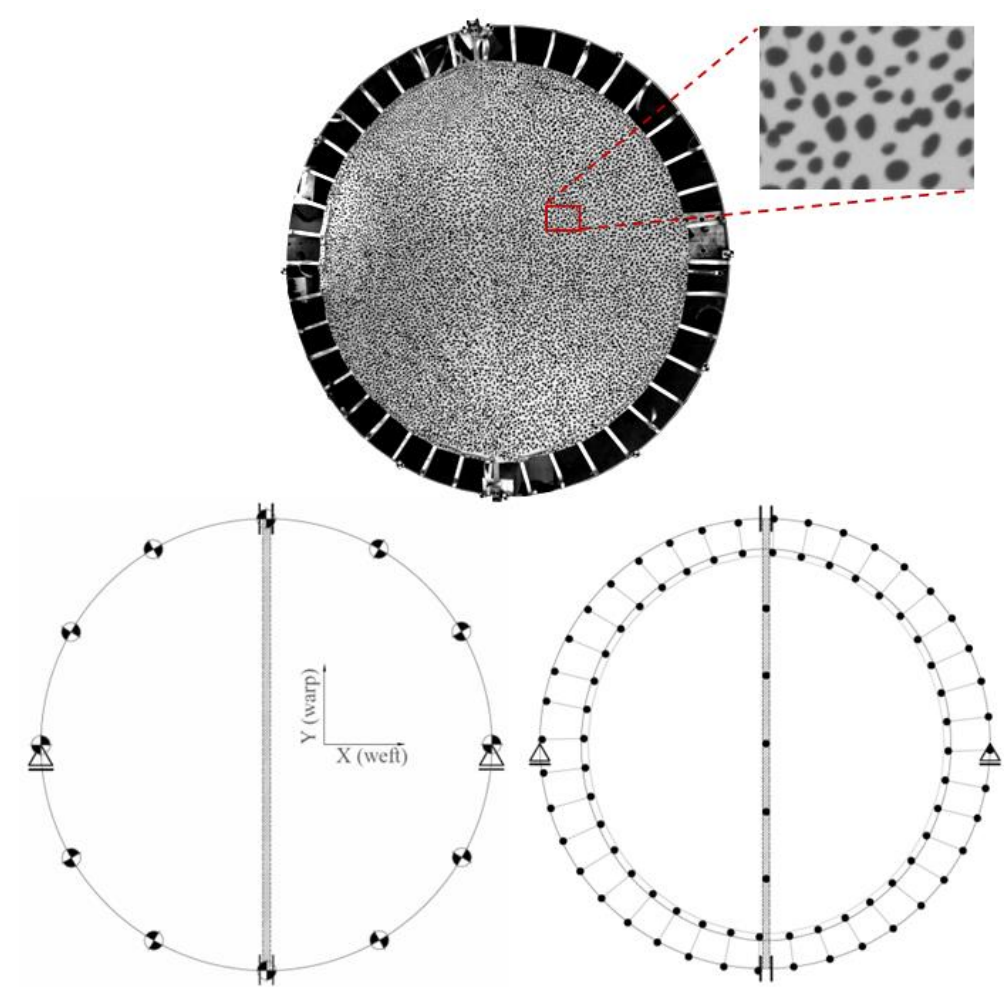

Figure 10. (top) A speckle pattern is applied on the membrane surface for full field DIC measurements; (left) A number of characteristic points are provided with markers and are measured throughout the test; (right) In the most important steps of the test, virtual markers are positioned for a more detailed geometry verification

\section{DIC ERROR ESTIMATION}

To estimate the error of the DIC measurements, a number of subsequent images are captured keeping the structure static. The small deformations and strains in the membrane give an indication of the error of the measurements for this specific set-up. Strain variations of $0.08 \%$ in weft (horizontal) and $0.07 \%$ in warp (vertical) direction and displacements of respectively $0.095 \mathrm{~mm}$ and $0.14 \mathrm{~mm}$ are measured.

\section{Numerical simulation}

In a preliminary parameter study [6] [11], the numerical design model is established through the real form-finding of a technical textile inside bending-active boundaries, where the applied prestress in the membrane determines the structure's shape (Figure 11 (top)). The model allows evaluating different design parameters to come to a structurally efficient kinematic textile hybrid, after which the cutting pattern can be derived to construct the obtained structural design.

The simulation process of the numerical experimental model, mimicking the process of the actual experiment, is fairly simple. First, the GFRP ring is modelled together with the internal beam element, 
incorporating the initial stresses in the ring due to the bending of a straight element. Secondly, the membrane's cutting pattern is generated and is connected to the GFRP ring by means of forty cable elements. The connection of the membrane to the internal beam element is realized using kinematic constraints, allowing the membrane to slide along the internal element. Finally, each of the links is assigned the length that is measured during the experiment at the form-finding state, which results in the creation of the three-dimensional pringle shape (Figure 11 (bottom)).

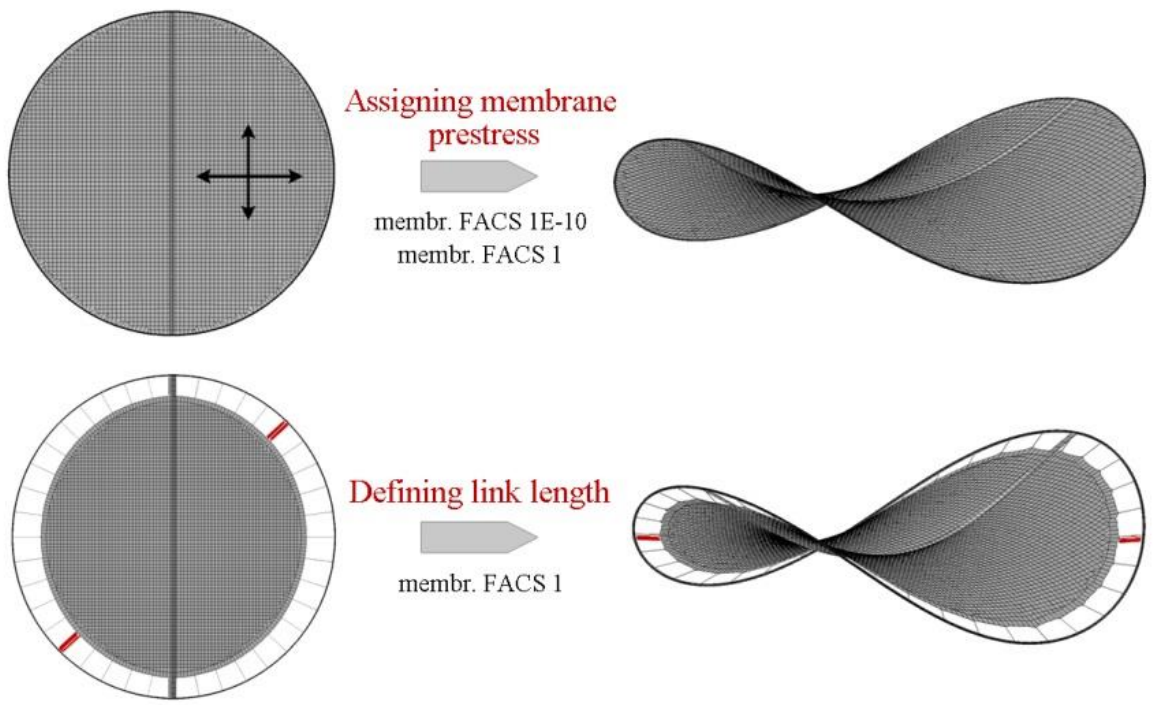

Figure 11. The 'numerical design model' vs. the 'numerical experimental model'

\section{Results and discussion}

This section describes the results of the experimental investigation and verifies the correspondence with the numerically obtained results. In the first place, the experimental results are compared to the 'numerical experimental model', i.e. the one mimicking the test process. In the next section (7), the accuracy of the 'numerical design model' is evaluated, providing information on the suitability and precision of the simulated design process.

\subsection{Overall geometry}

As described previously, the out-of-plane displacement of the pringle shape is established through the shortening of the forty polyester belts. The belts are tensioned bit by bit while their length is manually measured at different intervals, until the belts reach their intentional length of $15 \mathrm{~cm}$.

In the early phase of the pretensioning, i.e. when the small belts still have a length $>15 \mathrm{~cm}$, the structure is very unstable. As there is nearly no prestress in the membrane and no pronounced curvature is created yet, the structure can hardly carry its own self-weight (Figure 12 (left)). Slightly tensioning the contraction cable between the top points helps to stabilize the structure during this early stage of applying the prestress (Figure 12 (right)). From the moment the pretension is sufficient, this tensioning belt is removed in order to approach the actual prestressing process as close as possible. The comparison with the numerical simulation will only evaluate the final stages of the pretensioning (without the internal belt) and, of course, the kinetic deployment. 

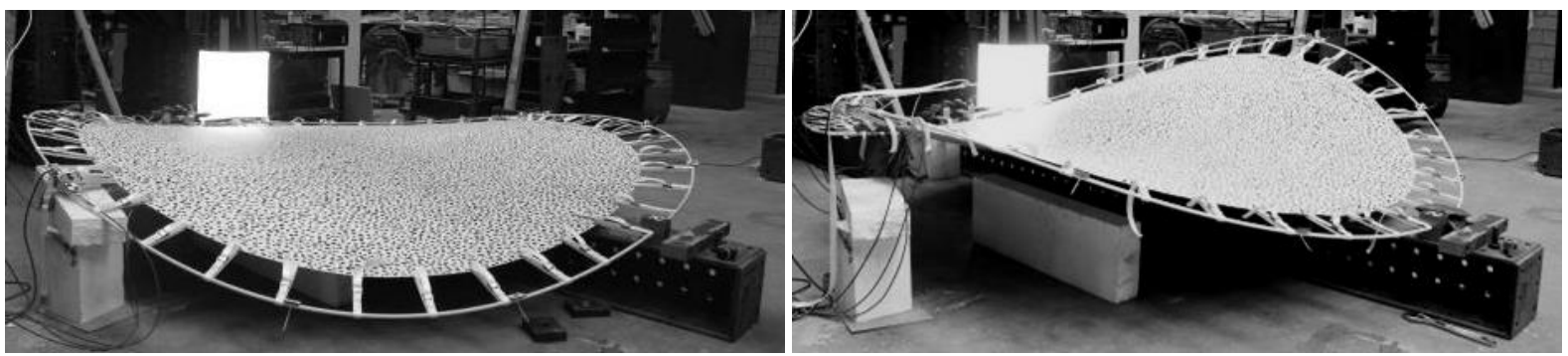

Figure 12. Instabilities in the early stage of the pretensioning: (left) asymmetric deflection of the structure; (right) slightly tensioning the belt between the top points helps stabilizing the structure during the early stage of the pretensioning

Figure 13 shows the textile hybrid in its fully tensioned state, i.e. when the belts have a length of \pm 15 $\mathrm{cm}$. In this state, the middle belt is removed and thus the structure is shaped solely through the prestressing of the membrane. Until this position, one of the lower points can slide horizontally along the steel beam. Afterwards, i.e. before the internal belt is shortened to simulate the kinetic deployment, this point is fixed by clamping the supporting point onto the beam.
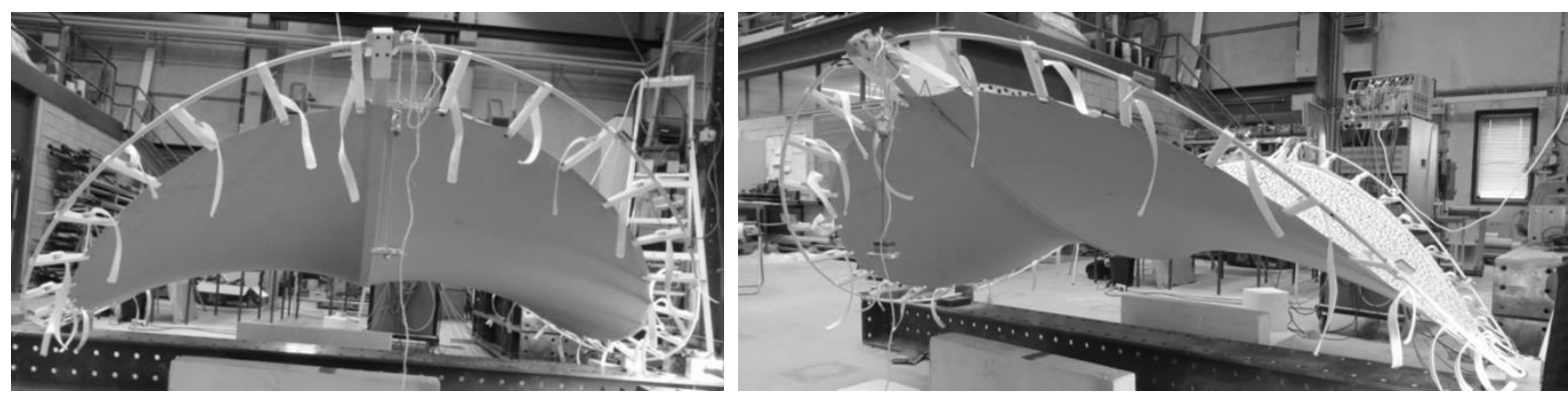

Figure 13. Three-dimensional geometry of the textile hybrid obtained by pretensioning the PU-membrane in the GFRP ring

The set-up of the DIC system allows measuring lengths and displacements in the three-dimensional space. Doing so for the points indicated in Figure 10 permits to verify the overall geometry of the experimental textile hybrid. Figure 14 (left) presents the experimentally obtained geometry after formfinding, i.e. when the links have a length of approximately $15 \mathrm{~cm}$ and Figure 14 (right) presents the obtained geometry when the contracting cable is shortened with a length of $30 \mathrm{~cm}$.

Comparing the geometries obtained in the experiment (Figure 14) and in the numerical experimental model (Figure 15), a relatively good correspondence is acquired. The out-of-plane movement of the top points is $88 \mathrm{~cm}$ for the experimental model and $90 \mathrm{~cm}$ for the numerical experimental model. The maximum deviation is observed in the distance between the top points, which is only $6.5 \mathrm{~cm}$ for a total distance of $267.5 \mathrm{~cm}$.

Those differences can be due to inaccuracies in the fabrication of the experimental model or set-up. The precision of the manually applied tensioning of the belts can also contribute to small inconsistencies and asymmetries. Furthermore, in the numerical model the lower points can slide freely in x-direction, whereas in the experimental model the friction between the support and the steel beam could obstruct this movement slightly. This could for example explain why the distance between the lower points is larger in the experimental than in the numerical model. 

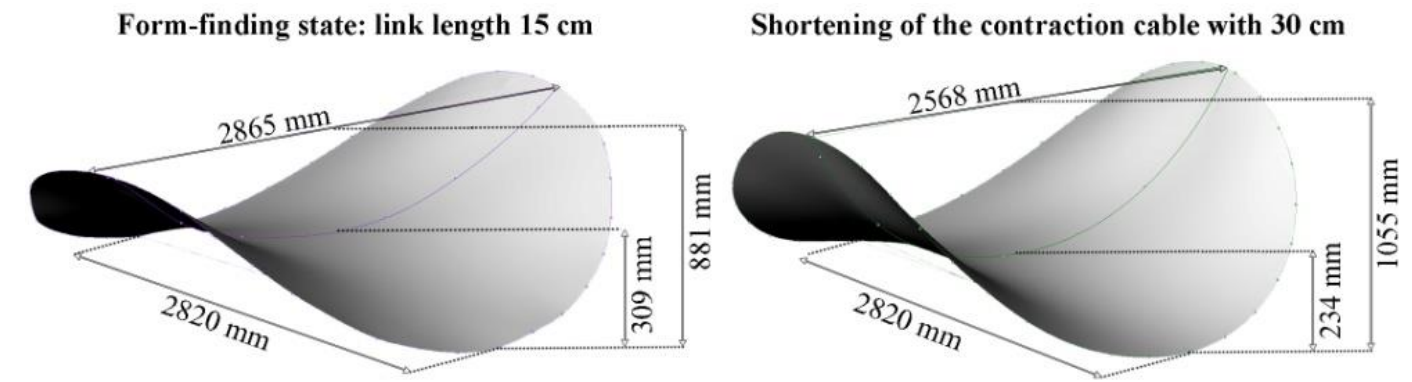

Figure 14. Geometrical parameters from the experimentally obtained structure: (left) after form-finding; (right) after contracting the internal cable

Form-finding state: link length $15 \mathrm{~cm}$

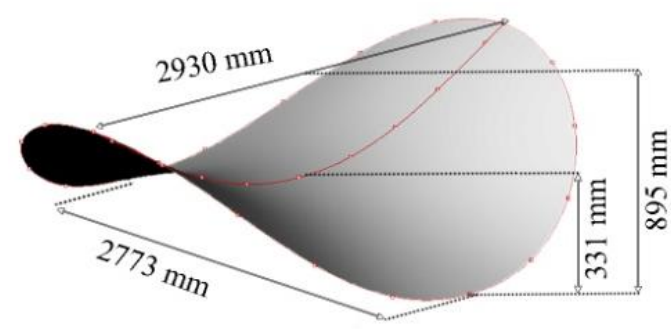

\section{Shortening of the contraction cable with $30 \mathrm{~cm}$}

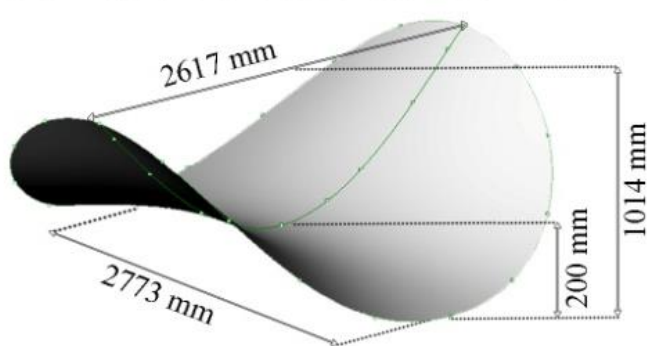

Figure 15. Geometrical parameters from the numerical results (numerical experimental model): (left) after "formfinding', i.e. when the belts have a length of approximately $15 \mathrm{~cm}$; (right) after contracting the internal cable

\subsection{Membrane Strains}

Furthermore, the strain distribution in the membrane surface is measured throughout the test by continuously tracking the applied speckle pattern with DIC, which allows visualizing the strains in horizontal (weft) and vertical (warp) direction. The unstressed flat membrane is taken as the reference state, i.e. where no strains are present. The resulting strain fields presented below are thus strains relative to this unstressed state: (i) after the form-finding (Figure 16) and (ii) after shortening the contraction cable with $30 \mathrm{~cm}$ (Figure 17).

It should be noted that the experimental results presented in Figure 16 and Figure 17 are projected on the undeformed reference geometry. As the DIC cameras are placed at a distance of $3.5 \mathrm{~m}$ away from each other, the angle whereby they capture the textile hybrid is relatively high. Consequently, the visualisation of the results on the out-of-plane deformed structure would give a false impression of the structure's deformations.

The two vertical strips where no correlation is obtained (in the experimental strain field) result from the tensioning belt between the top points that is integrated to keep the structure stable in the early phases of the pretensioning. The zones of the speckle pattern that are hidden by the contraction cable cannot be tracked and thus no strains can be calculated in those areas. Again, due to the angle of the cameras this results in two different hidden zones and thus in two vertical uncorrelated strips.

Looking at the experimental form-finding state (Figure 16 (top)), a zone with high straining of the membrane material in the vertical direction is located along the internal beam $\left(\boldsymbol{\varepsilon}_{\mathrm{yy}}=3.5 \rightarrow 4 \%\right)$, as a result of the beam pushing the membrane down. In this same zone, the strains in horizontal direction are smaller due to the effect of crimp interchange $\left(\varepsilon_{\mathrm{xx}}=0.2 \rightarrow 0.4 \%\right)$. In the central zone of each half (divided by the internal beam) the strains in warp and weft direction are quite similar (around 1.2\%). 
Along the membrane boundaries, in x-direction locally increased strain values are noticed around the top points, whereas for the y-direction locally increased strain values appear near to the lower points. This can be related to the (local) degree of curvature of the ring and thus its three-dimensional geometry.

The overall strain field resulting from the numerical experimental model (Figure 16Error! Reference source not found. (bottom)) looks quite similar to the experimental results. As the links of the numerical model are assigned the same length as in the experiment, also here small asymmetries are visible. In the centre of each half of the structure, the strains are slightly smaller in x-direction and at the same time slightly larger in y-direction than in the experimental model. This can be due to the friction of the sliding lower point during the 'form-finding' step. As mentioned in the previous section, the distance between the lower points is smaller in the numerical model, compared to the experiment. This could result in decreased strains in the numerical model in $\mathrm{x}$-direction $\left(\varepsilon_{\mathrm{xx}}= \pm 0.9 \%\right)$ and due to the effect of crimp interchange consequently in slightly increased strains in y-direction $\left(\varepsilon_{\mathrm{yy}}= \pm 1.3 \%\right)$.
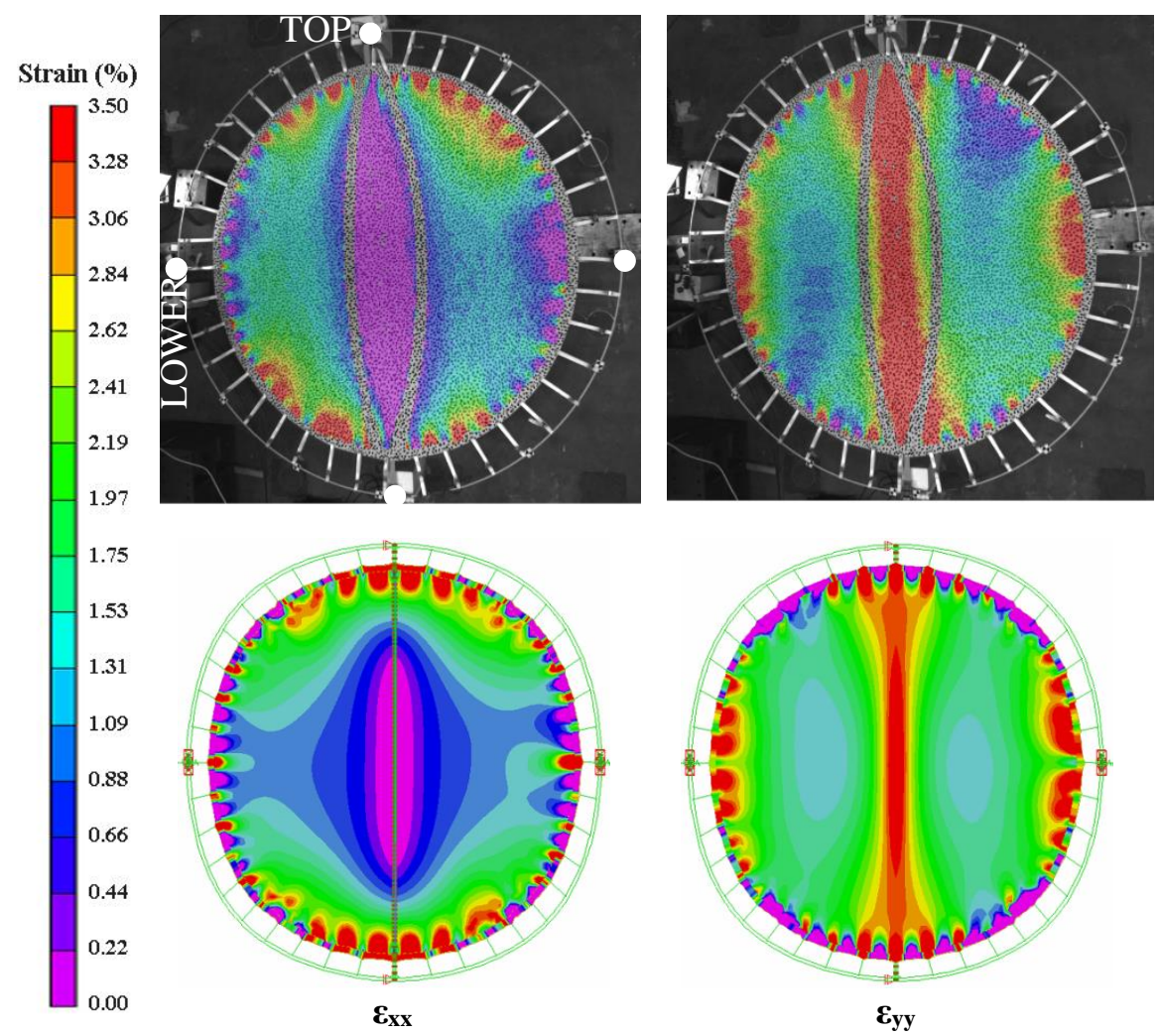

Figure 16. Strain fields (\%) after the 'form-finding': (top) experimental results in $\mathrm{x}$ - and $\mathrm{y}$-direction; (bottom) numerical results in $\mathrm{x}$ - and $\mathrm{y}$-direction

Next, the evolution of the strains is evaluated during the kinetic deployment, comparing the configuration where the contraction belt is shortened from $286.5 \mathrm{~cm}$ to $256.8 \mathrm{~cm}$ (i.e. $-30.3 \mathrm{~cm}$ ) (Figure 17).

Both in the experimental and the numerical experimental model, the strains in $\mathrm{x}$ - and $\mathrm{y}$-direction decrease when the top points are pulled towards each other. In the central zone of each half of the membrane, the strains in $\mathrm{x}$ - and y-direction drop to approximately $0.9 \%$ and $0.6 \%$ for the experimental and $0.6 \%$ and $0.1 \%$ for the numerical experimental model.

The overall structural response (when looking at the membrane strains) of the numerical simulation thus corresponds to the experimentally observed results, but when looking more in detail, some deviations 
are noticed. In x-direction, for example, the strains in the numerical model are indeed lower along the internal element, but they increase towards the middle of the internal beam, unlike in the experimental strain field.
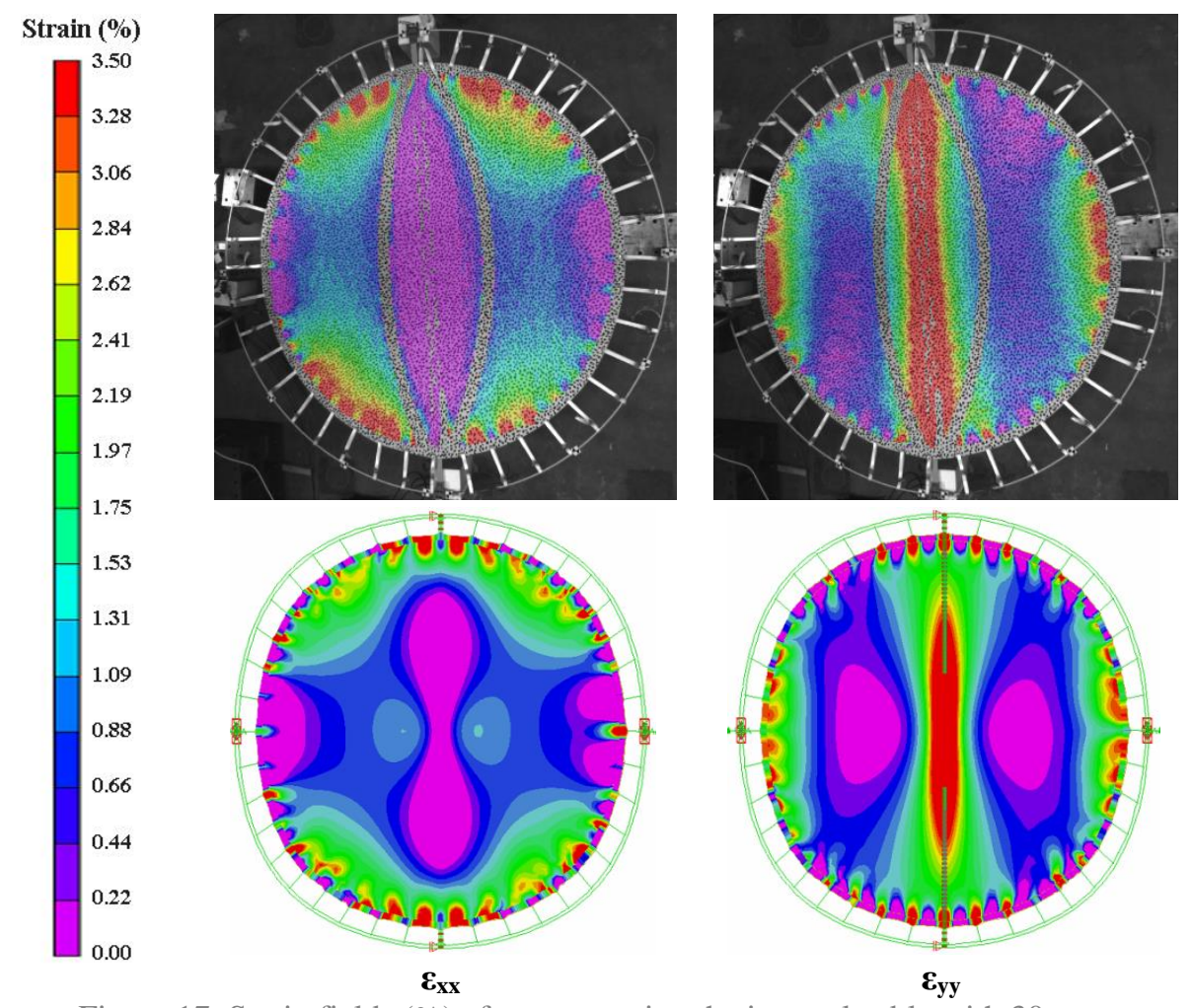

Figure 17. Strain fields (\%) after contracting the internal cable with $30 \mathrm{~cm}$ : (top) experimental results; (bottom) numerical results in $\mathrm{x}$ - and $\mathrm{y}$-direction

When comparing the geometry of the internal beam element it becomes clear why (see Figure 18): the beam in the numerical model is pushed down more in the middle, whereas the experimental beam approximates a more rounded shape. A possible explanation lies in the modelling of the interaction between the internal element and the membrane (by means of kinematic constraints in $\mathrm{x}$ - and $\mathrm{z}$ direction), which can differ from the actual experimental connection (e.g. due to the indeterminate friction).

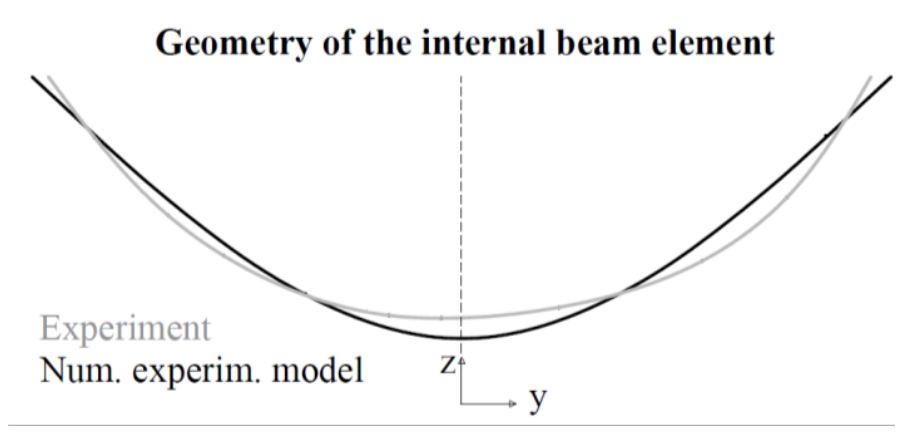

Figure 18. Geometry of the internal beam element with the cable shortened with $30 \mathrm{~cm}$ : experimental $(2568 \mathrm{~mm}$ between top points) vs. numerical model (2617 mm between top points)

After the kinetic deployment, the middle belt is again removed and the prestressing links are gradually loosened. The resulting strains in the membrane in this fully relaxed state are the permanent strains in the membrane (visualized in Figure 19). Logically, the permanent strains in warp direction (i.e. the y- 
direction) are smaller than the permanent strains in weft direction (i.e. the x-direction). A mean value of $0.68 \%$ in weft and $0.43 \%$ in warp direction are registered, which corresponds to the additional compensation factor (that needs to be applied besides the compensation that account for the elastic straining of the membrane material).

Comparing those values to the applied compensation factor of $4 \%$, one can conclude that the compensation was taken too high for this range of applied prestress and resulting forces. A tailored biaxial test should have been performed, similar to what is described in [11]. Unfortunately, the biaxial bench that is available at the Vrije Universiteit Brussel cannot cope with these low forces, as the load cells connected to this biaxial bench have a capacity of $100 \mathrm{kN}$ and thus a prestress of $0.3 \mathrm{kN} / \mathrm{m}$ lies within the noise range of the used load cells.

In this stage of the comparison (between the experiment and the numerical experimental model) this has no major influence, as this simulation is fully based on applied straining of the material. The 'formfinding' of the three-dimensional pringle shape is not obtained by imposing a certain prestress, but by shortening the belts, i.e. by applying a certain straining to the membrane's cutting pattern.

As this compensation factor of $4 \%$ (in both warp and weft direction) is applied to both the cutting pattern of the experiment and the numerical experimental model, no major deviations were expected in the strain field, nor in the geometry, due to this misconception on the material's actual compensation factor for this range of prestress (being $\pm 0.68 \%$ (x) and $\pm 0.43 \%$ (y), see Figure 19). Small deviations can however occur due to the approximated linear elastic behaviour that is used in the numerical models.

The reflection to the numerical design model, on the contrary, does not incorporate the influence of the compensation factor and will highlight the importance of applying a more correct/suited value. This comparison between the numerical experimental model and the numerical design model are discussed in section 7. Not only the strain fields are compared, but also the membrane forces and the overall geometry of the structure.

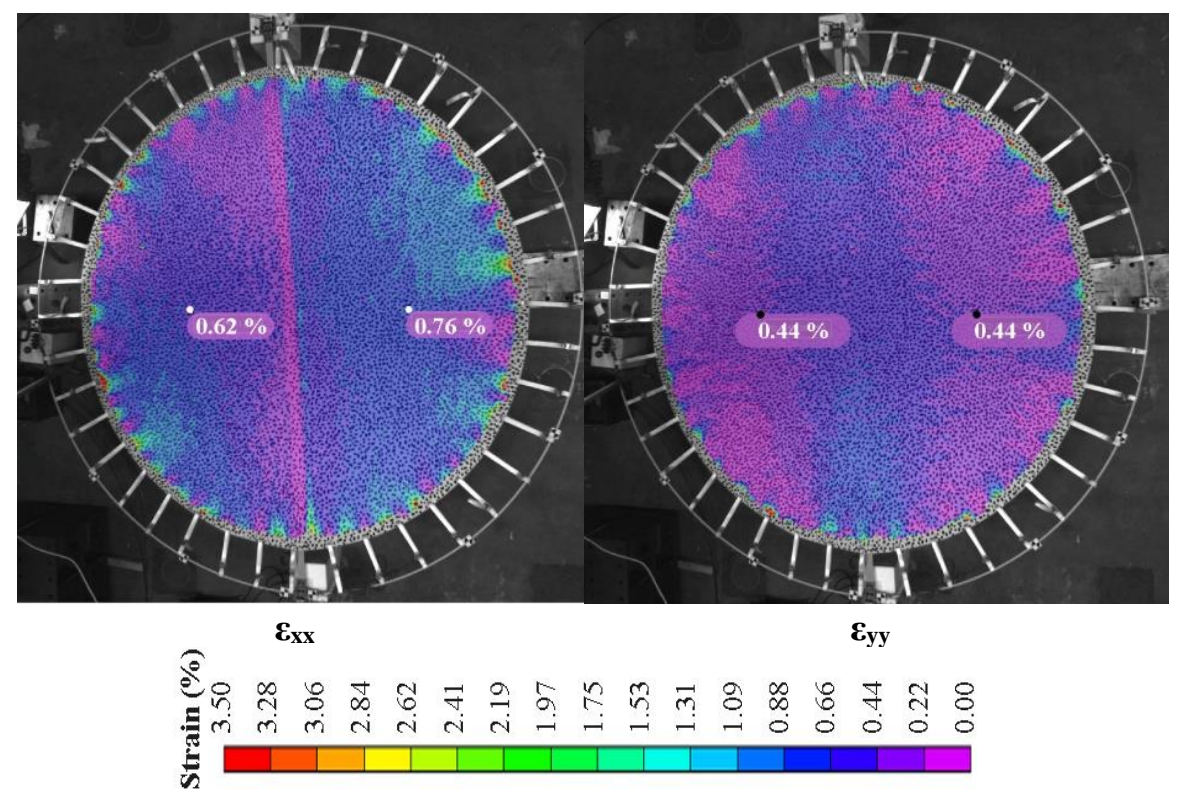

Figure 19. Strain fields (\%) after loosening the forty belts again, i.e. the permanent strain in the membrane material 


\subsection{Beam stresses}

The initial bending of the straight element towards a ring generates residual stresses in the ring of 163.5 $\mathrm{MPa}$, being the maximum stress on the circular section. This pure bending around the z-axis results in a distribution of the stresses along the section as presented in Figure 20 (left), reaching its maximum at point A. As the strain gauges are glued on the already deformed ring and, furthermore, are positioned on the top and bottom of the beam's section (where the stress resulting from this initial bending is zero, point B), these residual stresses are not captured by the strain gauges. Nevertheless, they should be taken into account when analysing the stresses in the beam element.

The actual out-of-plane deformation of the ring due to the pretensioning of the membrane, results in both bending in and perpendicular to the xy-plane (Figure 20 (left) and (right)). Superposition of the resulting stresses, consequently, leads to a shifted stress distribution on the section, where the maximum stress lies not on the position of the strain gauges.

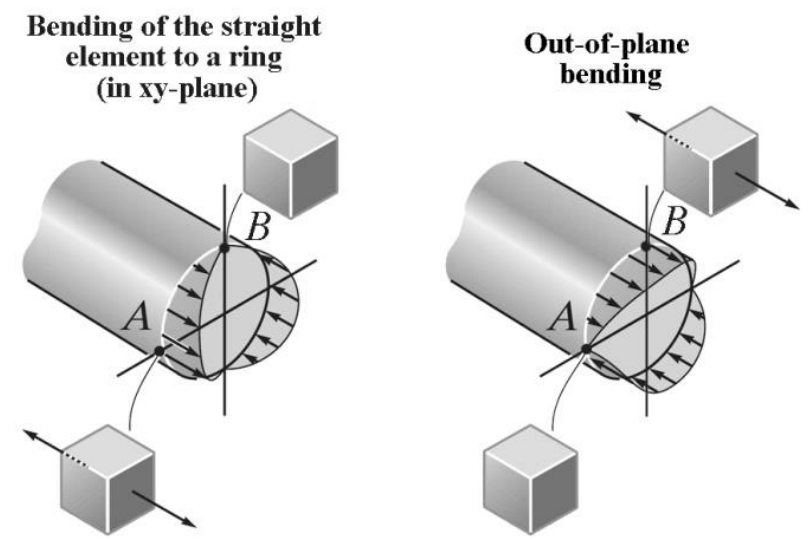

Figure 20. Distribution of stresses on a circular beam section: (left) bending in the xy-plane; (right) bending out of the xy-plane [based on figures from [12]]

Furthermore, due to the out-of-plane movement caused by adding the prestress and the kinetic deployment, the ring member rotates around its longitudinal axis. Therefore, the strain gauge rosette that is located at one of the top points is thus no longer positioned at the highest point of the beam section, but is rotated inwards (Figure 21).

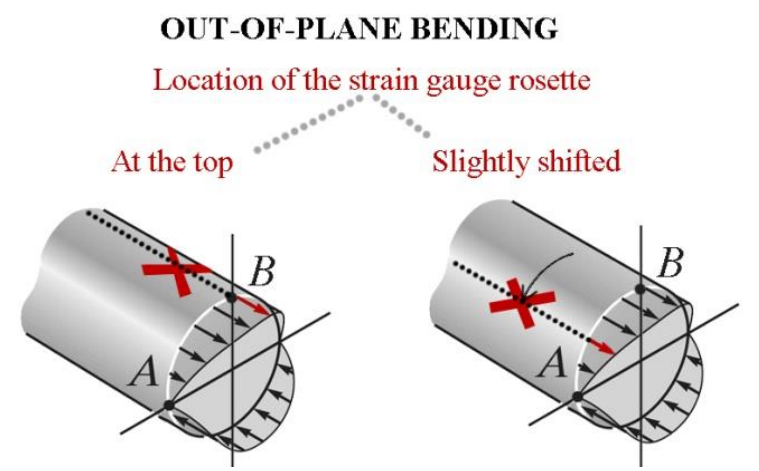

Figure 21. Due to the out-of-plane movement, the beam section is rotated around its longitudinal axis and lies no longer at the highest point of the section [based on figures from [12]] 


\section{EXPERIMENT}

During the experimental investigation, the strain gauge rosettes continuously register the strains in the GFRP ring. Each of the rosettes contains three strain gauges, oriented as indicated in Figure 22. Two strain gauge rosettes are positioned near one of the support points (on the upper side: 'Support UP', on the downward side: 'Support DOWN') and one rosette is positioned near one of the top points (on the upper side: 'High point UP').
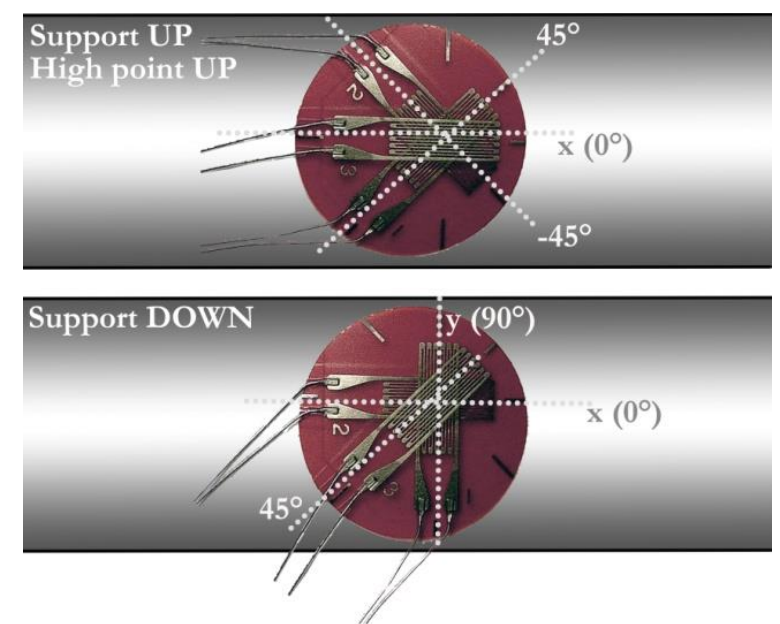

Figure 22. Orientation of the strain gauges; two strain gauge rosettes are positioned at one of the support points (on the upper and downward side) and one at one of the top points (on the upper side)

In function of the measured strains in the three directions $\left(\varepsilon_{1}, \varepsilon_{2}\right.$ and $\left.\varepsilon_{3}\right)$ and the orientation angle of the strain gauges $\left(\theta_{1}, \theta_{2}\right.$ and $\left.\theta_{3}\right)$, the normal strains $\left(\varepsilon_{x}\right.$ and $\left.\varepsilon_{y}\right)$ and the shear strain $\left(\gamma_{x y}\right)$ can be calculated, combining the following formulas [12]:

$$
\begin{aligned}
& \varepsilon_{1}=\varepsilon_{x}\left(\cos \theta_{1}\right)^{2}+\varepsilon_{y}\left(\sin \theta_{1}\right)^{2}+\gamma_{x y} \sin \theta_{1} \cos \theta_{1} \\
& \varepsilon_{2}=\varepsilon_{x}\left(\cos \theta_{2}\right)^{2}+\varepsilon_{y}\left(\sin \theta_{2}\right)^{2}+\gamma_{x y} \sin \theta_{2} \cos \theta_{2} \\
& \varepsilon_{3}=\varepsilon_{x}\left(\cos \theta_{3}\right)^{2}+\varepsilon_{y}\left(\sin \theta_{3}\right)^{2}+\gamma_{x y} \sin \theta_{3} \cos \theta_{3}
\end{aligned}
$$

Following the material property relationships, also the normal and shear stresses can be calculated, by means of the following equations:

$$
\begin{gathered}
\varepsilon_{x}=\frac{\sigma_{x}}{E}-\frac{v \sigma_{y}}{E} \\
\varepsilon_{y}=\frac{\sigma_{y}}{E}-\frac{v \sigma_{x}}{E} \\
\gamma_{x y}=\frac{\tau_{x y}}{G}
\end{gathered}
$$

The results measured during the experimental investigation are summarized in Table 2. Based on the normal and shear stresses, also the Von Mises stresses are calculated (used for the comparison with the numerical model):

$$
\sigma_{\text {Von Mises }}=\sqrt{\sigma_{x}^{2}-\sigma_{x} \sigma_{y}+\sigma_{2}^{2}+3 \tau_{x y}^{2}}
$$


Table 2. Resulting strains and stresses from the strain gauge rosettes: Rosette $1=$ support, top; Rosette $2=$ support, bottom; Rosette 3 = top point, top

\begin{tabular}{|c|c|c|c|c|c|c|}
\hline & \multicolumn{3}{|c|}{$\begin{array}{l}\text { Form-finding } \\
\quad \text { PIC728 }\end{array}$} & \multicolumn{3}{|c|}{$\begin{array}{c}\text { Cable contraction by } 30 \mathrm{~cm} \\
\text { PIC } 873\end{array}$} \\
\hline & Rosette 1 & Rosette 2 & Rosette 3 & Rosette 1 & Rosette 2 & Rosette 3 \\
\hline$\varepsilon_{x}(\mu$ strain $)$ & -4299 & -1151 & 1204 & -4974 & -1372 & 2285 \\
\hline$\varepsilon_{y}$ ( $\mu$ strain) & 1579 & 4220 & -458 & 1668 & 4664 & -779 \\
\hline$\gamma_{x y}(\mu$ strain) & -742 & -1469 & 123 & -548 & -865 & -477 \\
\hline$\sigma_{x}(\mathrm{MPa})$ & -170.4 & -7.8 & 47.6 & -198.7 & 12.9 & 91.2 \\
\hline$\sigma_{y}(\mathrm{MPa})$ & 25.7 & 171.2 & -7.83 & 22.7 & 188.2 & -11.0 \\
\hline$\tau_{x y}(\mathrm{MPa})$ & -12.4 & -24.5 & 2.1 & -9.13 & 14.4 & -8.0 \\
\hline Von Mises (MPa) & 186 & 180 & 52 & 212 & 197 & 98 \\
\hline
\end{tabular}

\section{NUMERICAL EXPERIMENTAL MODEL}

From the numerical experimental model the Von Mises stresses along the cross section can be visualized. An example of the distribution near to the support point (at the location of the strain gauge rosettes) is given in Figure 23 for the situation where the contraction cable is shortened with $30 \mathrm{~cm}$.

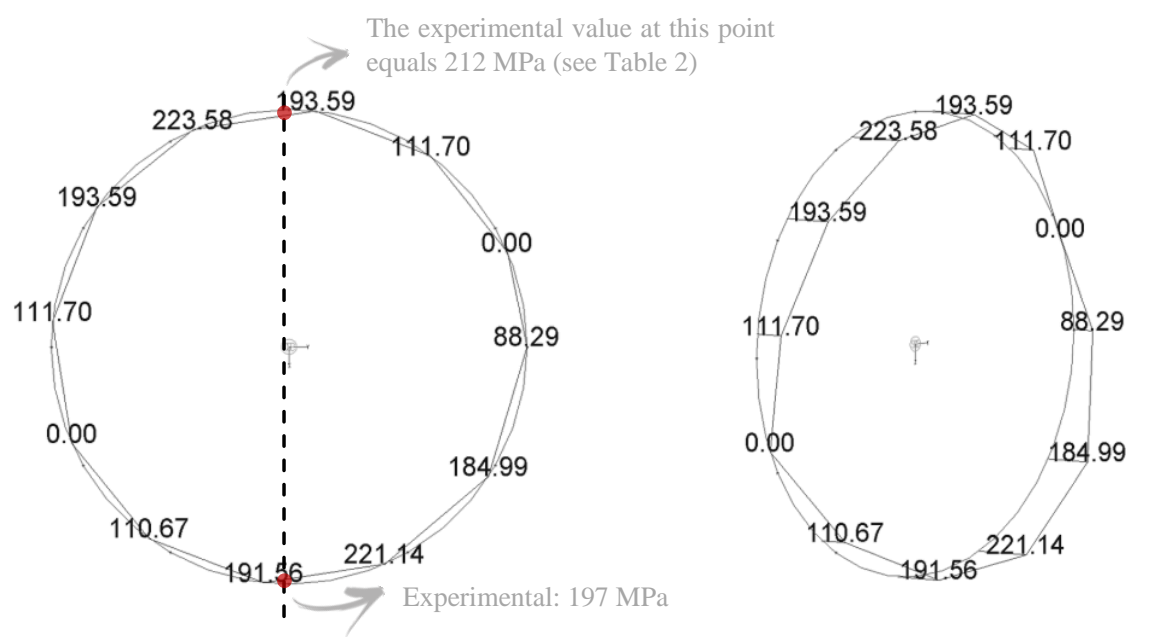

Figure 23. Distribution of the Von Mises stresses (MPa) on the ring section in Sofistik: example at the position of the strain gauges near the support point when the contraction cable is shortened with $30 \mathrm{~cm}$ (left: front view, right: 3D view)

As the results from the Sofistik simulation are not always presented at the exact location of the strain gauge rosette (in Figure 23 at the top and bottom of the section), the needed value is obtained by interpolation between the two adjacent values.

An overview of the obtained Von Mises stresses at the location of the strain gauge rosettes for both the 'form-finding' configuration and the deployed situation ( $30 \mathrm{~cm}$ contraction) is given in Table 3 . Comparing those to the values presented in Table 2 shows a relative good correspondence for both the form-finding and the contracted configuration. Near the lower point slightly higher values of Von Mises stresses are obtained in the numerical model, compared to the experimentally obtained values. Looking into the Von Mises stresses in the top point, larger deviations between experiment and numerical model 
are observed, which can be due to the approximated location. For all strain gauge rosettes an increase in stress is noted when contracting the cable element.

Table 3. Von Mises strains at the positions of the strain gauge rosettes: Rosette $1=$ support, top; Rosette $2=$ support, bottom; Rosette 3 = top point, top

Form-finding

\section{Rosette 1}

Von Mises (MPa)
191
Rosette 3

188
Cable contraction by $30 \mathrm{~cm}$

$\begin{array}{ccc}\text { Rosette } 1 & \text { Rosette } 2 & \text { Rosette } 3 \\ 200 & 198 & 82\end{array}$

\section{Validation of the numerical design model}

In the previous section, the experimental results are evaluated and compared to the results from the numerical experimental model. However, this model does not allow to properly re-design the structure by changing design parameters, since the membrane's cutting pattern is fixed and no actual form-finding is performed in this numerical process (i.e. with FACS 1E-10 in Sofistik). This model rather creates new equilibrium shapes with the same structural elements. It is thus important to also reflect on the initial numerical design model, whereby this cutting pattern is created.

As was already initiated in section 6.2, it is expected that the cutting pattern used for the numerical experimental model is too much compensated, especially in the warp direction (y). The comparison of geometry and stress and strain patterns between the 'numerical design model' and the 'numerical experimental model using the overcompensated cutting pattern', confirms this prediction (Figure 24). However, due to the great interaction between the ring and the membrane stresses, the effect of this overcompensation is not straightforward. Intuitively, a smaller cutting pattern would result in higher strains and higher membrane forces, but due to the large beam deformations this holds not true, as can be seen in Figure 24. Furthermore, the significant importance of crimp interchange results in a decrease in strain in $\mathrm{x}$-direction as the strain in y-direction increases.

Along the internal beam element the strains and forces in y-direction are indeed higher, compared to the numerical design model. In the $\mathrm{x}$-direction on the contrary, the stresses and strains are remarkably lower than predicted in the numerical design model. Unlike in the y-direction, where the inward movement of the upper points is partially limited due to presence of the internal beam element, the lower points can move freely in $x$-direction. Due to the higher pretension (due to the smaller cutting pattern) the lower points are pulled more inwards, resulting in the larger deformation of the ring element and consequently in lower stresses and strains in a big part of the membrane area (Figure 24). Note that it was already discussed in [6] and [11] that the larger the deformations (due to a higher pretension), the less homogeneous the stress distribution is. Together with the effect of crimp interchange, the stresses and strains in $\mathrm{x}$-direction along the internal beam are thus remarkably lower than in the numerical design model.

Looking at the geometry, the numerical experimental model indeed shows higher deformations than the numerical design model: the height of the top points lies $83 \mathrm{~mm}$ higher, the distance between the top points is $78 \mathrm{~mm}$ shorter and the lower points lie $47 \mathrm{~mm}$ closer to each other.

Therefore, the cutting pattern of the membrane is re-calculated, integrating additional compensation factors, i.e. the part of the compensation that takes into account the permanent deformation of the membrane, of $0.43 \%$ and $0.68 \%$ in respectively warp and weft direction (as determined from the 
experimental investigation). An adapted numerical experimental model is investigated using this new cutting pattern.

Figure 24 compares the results of this adapted numerical experimental model with the numerical design model. Especially in the warp direction (i.e. the y-direction) significant improvements are observed. The high stresses and strains along the internal beam have decreased considerably, whereby the overall stress distribution and strain pattern lies much closer to the ones of the numerical design model (compared to the original numerical experimental model). Although also the distributions in $\mathrm{x}$-direction correspond better, still a larger difference with the numerical design model is observed compared to the distributions in y-direction.

Concerning the geometry of this adapted numerical experimental model with a modified cutting pattern, other improvements are obtained: the difference in distance between the top points drops to $28 \mathrm{~mm}$, the difference in distance between the lower points to $2 \mathrm{~mm}$ and the difference in height to $20 \mathrm{~mm}$.

It can be concluded that the performance of a tailored biaxial test in order to determine the appropriate compensation factors is of major importance. Considering this, the numerical design model can be used to design and analyse the structural behaviour of the kinematic textile hybrid.

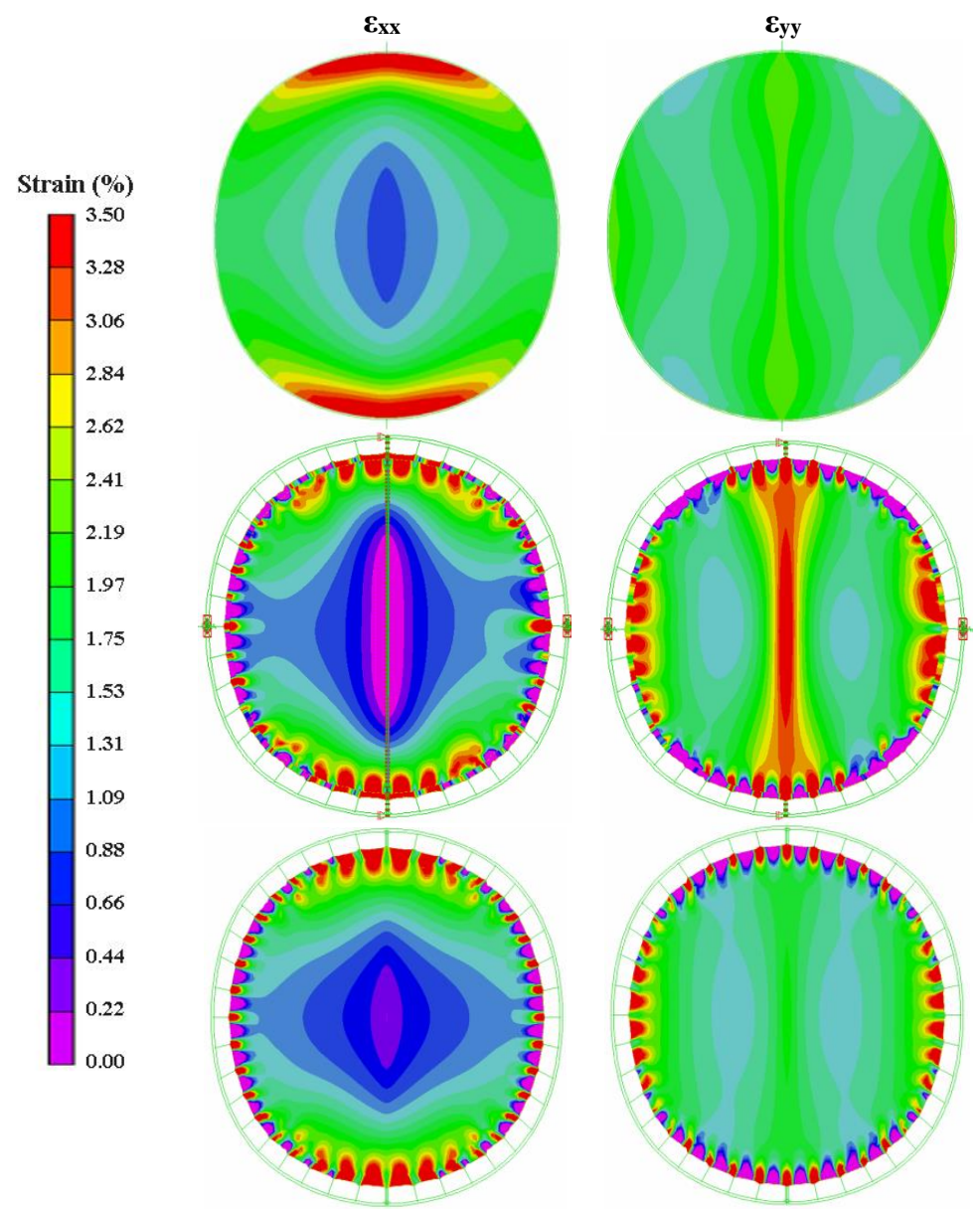

Figure 24. Comparison of the strain distribution (\%) for the 'numerical design model' (top), the 'numerical experimental model' (middle) and the 'adapted experimental model', i.e. with adapted cutting pattern (bottom) 


\section{Conclusions}

The experimental investigation confirms the feasibility of designing kinetic textile hybrids. The analysed principles of both the prestressing (and form-finding) of the membrane and the kinetic deployment of the structure are tested and evaluated. Thanks to the experimental study a lot of insight is gained in the behaviour of the structure, which was very valuable for the further structural investigation.

The comparison of the experiment with the numerical experimental model shows good correspondence. The geometries, strain field patterns and measured beam stresses of the numerical experimental model lie close (within an acceptable range) to the experimental results. Using a linear elastic orthotropic material model allows thus to predict the overall structural behaviour of the textile hybrid. Nevertheless, some small deviations are present (mostly) due to the inaccuracies in the experimental model.

Furthermore, the results of the numerical experimental model are compared to the numerical design model, in order to validate if the adopted form-finding process for the design of textile hybrids can predict the actual behaviour. The residual strains in the membrane (i.e. the permanent deformation) after the experimental investigation already indicate that the adopted compensation factor (= elastic strain + permanent strain) is taken too high. The analysis of the numerical experimental model and the numerical design model confirms this presumption. Therefore, an adapted numerical experimental model (with a modified cutting pattern) is established and analysed, resulting in a structural response comparable to the numerical design model. Considering the major importance of defining the correct compensation factor, this numerical design method can be used for the structural analysis of textile hybrids.

\section{Acknowledgements}

This PhD-research was conducted with the funding of the Research Foundation Flanders (FWO). Furthermore, the researchers would like to thank Sioen Industries for providing fabric material for the experimental investigation and the COST-Action TU1303 Novel Structural Skins for the possibility to join WG5 Materials and analysis.

\section{References}

[1] Images from https://senatus.net

[2] Images from http://www.str-ucture.com

[3] Lienhard, J., 2014. Bending-active structures: form-finding strategies using elastic deformation in static and kinetic systems and the structural potentials therein. Stuttgart, Germany: Institut für Tragkonstruktionen und Konstruktives Entwerfen (ITKE), Doctoral thesis.

[4] Lienhard, J., Riederer, J., Jungjohann, H., Oppe, M., Knippers, J., 2013. Multifunctional adaptive façade at IBA 2013; Design studies for an integral energy harvesting façade shading system, VI International Conference on Textile Composites and Inflatable Structures. 
[5] Images from http://www.kvarch.net/ and http://www.iba-hamburg.de/

[6] Puystiens, S., Van Craenenbroeck, M., De Laet, L., Van Hemelrijck, D., Van Paepegem, W., Mollaert, M., Implementation of bending-active elements in kinematic form-active structures - Part I: Design of a representative case study. Composite Structures (submitted)

[7] Sofistik AG. Software for better Design. http://www.sofistik.com

[8] Galliot, C. \& Luchsinger, R. H., 2009. A simple model describing the non-linear bi-axial tensile behaviour of PVC-coated polyester fabrics for use in finite element analysis. Composite Structures, 90(4), pp. 438-447.

[9] Correlated Solutions. Principle of Digital Image Correlation. http://www.correlatedsolutions.com/digital-image-correlation/

[10] Sioen Industries. Coated technical textiles - Architectural Membranes. http://www.sioen.com/

[11] Puystiens, S., 2017. Kinematic form-active structures for architectural applications: design, analysis and experimental verification, Vrije Universiteit Brussel, Brussels, Belgium, PhD thesis.

[12] Hibbeler, R., 2011. Mechanics of Materials. 8th ed. United States of America: Pearson Prentice Hall. 